\title{
The Platforms of Podcasting: Past and Present ${ }^{1}$ John L. Sullivan
}

\begin{abstract}
This article explores the role of digital platforms in podcasting (both past and present) and their impacts on the emergent podcast industry structure, content, and governance. Nieborg and Poell's (2018) theoretical framework for understanding the impacts of platformization on culture is leveraged here to better understand the changes underway in podcasting. Like other forms of media, podcasting is being profoundly re-shaped by platformization, though these transformations are distinct from other media in several key ways. Because podcasting emerged as a technology at the beginning of the 21st Century before the advent of social media and the cloud, its decentralized architecture is structured around RSS, also known as "Really Simple Syndication." When Apple added RSS aggregation into their iTunes Music Store in 2005, their market dominance in digital audio sales shaped early popular conceptions for the medium. I then outline how platformization is reshaping podcasting today by exploring how the three primary functions of media-related platform services - storage, discovery, and consumption - are shaping producers' and audience experiences. Market imperatives for audience consumption data, as well as the structural features of platforms, are currently fueling industry consolidation. Even though podcasting is built upon the open architecture of RSS, commercial pressures and the desire of market players to capitalize on the "winner-takeall" features of platforms are shaping the trajectory of the medium's current development.
\end{abstract}

\section{Keywords}

podcasting, platform, Apple, RSS, distribution, audio, media industries

This article has been accepted for publication in the journal Social Media + Society. Please carefully note that subsequent versions of this manuscript may have different content.

Podcasting is expanding rapidly as a popular cultural phenomenon, connecting listeners to audio content created by professionals, radio stations, and amateur hobbyists. Recent data from Edison Research revealed that an estimated 73 million Americans had listened to a podcast in the previous month, and those audiences listened to an average of seven podcasts per week (Edison Research, 2018). Encouraged by the huge success of the 2014 podcast sensation Serial - which was downloaded more than 80 million times in the first six months (Mallenbaum, 2015) entrepreneurs and legacy media companies with commercial interests in broadcasting have rapidly expanded their commercial interests in podcasting, bringing professional standards and the logics of capital with them. The increased visibility of podcasting in the past ten years is due in no small measure to the market power Apple's digital platform (specifically its Apple Podcasts directory, iOS mobile operating system, and hardware such as iPods and iPhones). For example, podcast hosting firm Blubrry (2017) reported that approximately $56 \%$ of all podcasts it hosted in 2017 were downloaded or accessed via Apple's platform. After years of requests, in late 2017 Apple

\section{Corrresponding Author:}

John L. Sullivan, Muhlenberg College, Dept. of Media and Communication, Allentown, PA 18104, USA.

Email: johnsullivan@muhlenberg.edu

1 The author would like to gratefully acknowledge the Faculty Development and Scholarship Committee as well as the Provost for providing funds to support this research. 
"This essay explores the role of digital platforms in podcasting (both past and present) and their impacts on the emergent podcast industry structure as well as its content and form." began releasing some limited forms of podcast consumption data to its users (Kafka, 2017; Webster, 2017). Other major tech companies such as Google and Spotify have also recently integrated podcasting into their their own existing music services, further expanding the potential reach of the medium. Podcast hosting firms such as Stitcher, Libsyn, blubrry, and Podbean (among others) have also become important players in the podcasting ecosystem by lowering offering ancillary production and data analytical services to podcasters.

This essay explores the role of digital platforms in podcasting (both past and present) and their impacts on the emergent podcast industry structure as well as its content and form. As suggested by Srnicek (2016, p. 43), the key leverage provided by platforms stem from the ability of these services to "monopolize, extract, analyze, and use the increasingly large amounts of data that [are] being recorded." In the case of podcasting, the market imperative for audience consumption data, in particular, is fueling industry consolidation among these competing platforms, though Apple's early dominance makes it the most likely beneficiary of podcast platformization. The increased centrality of platforms like Apple Podcasts, Spotify, and Google Podcasts will make it more likely that discovery takes place via these platforms. These major distribution platforms are increasingly operating as gatekeepers to audiences by canalizing audience attention into a smaller number of high-profile shows. As I note in the conclusion, podcasting's early roots as a service built upon the open architecture of RSS would seem to blunt the forces of platformization gripping other forms of online media. However, commercial pressures and the desire of market players to capitalize on the "winner-take-all" features of platforms may begin to take a more active role in shaping the trajectory of the medium's current development.

\section{Podcasting and Platformization}

Platforms can be understood at their most basic level as "digital infrastructures that enable two or more groups to interact" (Srnicek, 2016, p. 43). These infrastructures act as intermediaries between different types of users, including customers, suppliers, producers, service providers, suppliers, and advertisers. Gillespie (2018, p. 254) offers a similar definition, noting that platforms can be understood as "sites and services that host public expression, store it on and serve it up from the cloud, organize access to it through search and recommendation, or install it onto mobile devices". Platforms consist both of the technical infrastructure that allow for sharing of information as well as a set of rules (governance) that enable and constrain particular types of user activity. The shared technical infrastructure and open nature of these platforms ${ }^{2}$ often provides them with the aura of neutrality, much like a utility or common carrier service. Platforms are much more than neutral arbiters of interactions and transactions, however. By shaping the types of interactions among their participants in differential ways, platforms can also shape "how modularity and power are negotiated between a core unit with low variability and heterogeneous components of high variability" (Plantin, Lagoze, Edwards, \& Sandvig, 2018, p. 298).

In a Web 2.0 era, platforms have become a focal point for scholarly inquiry due to

2 Platforms are "open" in the sense that they enable users to easily create, store, and distribute media content with little to no technical knowledge. This "openness" is bounded in important ways, however. For example, users typically have little control over the storage or modification of their data. Additionally, the algorithms that make their content available to other platform users are often opaque at best. 
their increased centrality in the creation and distribution of media. The financial success of major sites like YouTube, Facebook, and iTunes has encouraged the proliferation of new platforms in the marketplace. Nieborg and Poell (2018, p. 2) have identified these changes as platformization, or "the penetration of economic, governmental, and infrastructural extensions of digital platforms into the web and app ecosystems, fundamentally affecting the operations of the cultural industries." Their model emphasizes three specific aspects of platformization: how platforms shift larger market structures, how cultural production is governed through platforms, and how platformization transforms the infrastructure of cultural production (via data-oriented practices such as algorithms, data structures, SDKs, and APIs, among others.). This last element, they note, refocuses attention on the "actual production and circulation practices" that are both enabled and constrained by platforms (Nieborg \& Poell, 2018, p. 8). Nieborg and Poell's theoretical framework is particularly useful for understanding the impacts of platformization on culture, and is leveraged here to better understand the changes currently underway in podcasting. Like other forms of media, podcasting is also being re-shaped by platformization, though these transformations are distinct from other types of media largely due to the openness of its technical infrastructure.

\section{An Open Architecture: RSS as Anti- Platform}

Podcasting can be understood as "a technology used to distribute, receive, and listen, ondemand, to sound content produced by traditional editors such as radio, publishing houses, journalists, and educational institutions...as well as content created by independent radio producers, artists, and radio amateurs" (Bonini, 2015, p. 21). One of the distinguishing features of podcasting is the openness of its distribution mechanism. Because it emerged as a technology at the beginning of the 21st Century before the advent of social media and the cloud, podcasting features a decentralized technical architecture, whereby audio content is stored all over the web and linked together via RSS, also known as "Rich Site Summary" (or, "Real Simple Syndication"). RSS was initially developed by Dan Libby and Ramanathan V. Guha at Netscape in 1999 as a text-based tool for allowing users to get automatic updates from blogs and other websites. As an open technical standard, RSS is free and allows listeners (via a helper app or "podcatcher") to locate, subscribe, and listen to new content without the necessity of visiting a specific storage platform or website (Markman \& Sawyer, 2014, p. 20).

Given its later significance in the popularization of podcasting, it is worth noting that the seeds of RSS were originally sown within Apple itself. Guha had developed a precursor software project in 1995 called the Meta Content Framework (MCF) while he was a software developer at Apple. MCF was intended to function as a means to describe and catalog metadata about webpages, but when Steve Jobs returned to Apple in 1997 as its CEO, he shut down much of Apple's research activity.

Guha then began developing a new version of MCF at Netscape called the Resource Description Framework (RDF) which was based in the new XML format (Hammersley, 2003, p. 2). Netscape popularized the first use of RDF Site Summary (RSS) in its browser by displaying headlines and links from other web pages within 
a single window, something that was unique to its browser.

RSS was eventually abandoned at Netscape and left to the developer community. Dave Winer, CEO of Userland software, released RSS version 0.91, though this competed with a more complicated and enhanced version of the software (RSS 1.0) championed by Rael Dornfest at O'Reilly (Hammersley, 2003, p. 4). The innovation of RSS feeds for podcasting occurred in 2000 when Dave Winer discussed with former MTV VJ Adam Curry the possibility of leveraging web content syndication for video, though the technology would be later implemented with audio files (Cochrane, 2005b, p. 8). Winer developed the concept of "enclosure" whereby URL addresses could be passed along to an aggregator. In September 2003, Winer created a feed with enclosures and married it to a software script he wrote called "iPodder" that would move MP3 files from Userland Radio's website to Apple's iTunes software (Wikipedia, 2017) and finally to his own iPod for listening. Curry's iPodder was the first podcatcher to leverage RSS feeds to allow for syndicated audio web content, or podcasting. The addition of media enclosures to RSS by software developer Dave Winer and Adam Curry allowed for media aggregators to locate and create directories for web content via the feed (For a full discussion of the early history of internet audio, see Berry, 2006; Bottomley, 2016; Sterne, Morris, Baker, \& Freire, 2008). The word "podcasting" wasn't popularized until 2004, when journalist Ben Hammersley (2004) coined the term in an article in The Guardian (he also suggested the term "audioblogging").

Unlike many platform-based cultural forms available on the web today (such as YouTube for video, Flickr for photos, and Facebook for social data, for example) wherein the platform acts as a centralized repository of data through which it connects producers and audiences, podcasting data files are scattered across the internet and connected together via RSS links. Key to the early popularization of the podcasting was the ability of programmers like Winer and Curry to leverage the open RSS standard to write new code (iPodder) that would enhance its features by allowing it to interface with existing audio playback software (iTunes) and hardware (iPods).

\section{Apple's iTunes and Early Podcast Platformization}

Podcasting has been inextricably linked to tech giant Apple, which was chiefly responsible for popularizing it in the early 2000s. In the first few years of its existence, users were required to cut and paste RSS links into podcatching software in order to download audio files and syndicate (or "subscribe to") a podcast. In an era before social media giants like Facebook and Twitter, RSS feeds were popularized via individual websites, through blogs, traditional news websites, and via several new online directories that were launched in 2005. Two of the most popular directories of the time were PodcastAlley.com and PodcastPickle.com (Cochrane, 2005a). Thematic podcast networks also began to emerge, such as techpodcasts.com, which featured technologythemed podcasts. Users during this era were required to utilize a multi-step process to successfully listen to a podcast: first, locate the podcast via one of these small directories; second, copy the RSS feed address; third, paste it into podcatcher software; and finally, download the audio file to the computer for playback.

While RSS made it technically possible for users to subscribe via podcatcher software like iPodder, the process was cumbersome and 
not well understood outside communities of tech enthusiasts. When Apple CEO Steve Jobs announced the release of iTunes 4.9 at the Apple Worldwide Developers Conference on June 28, 2005, he trumpeted its ability to provide easy access to audio podcasts, calling podcasting "TiVO for radio." Unlike iTunes' music store, which sold music MP3 files direct to consumers, iTunes operated as a visually attractive and easily-navigable podcatcher, allowing users to subscribe to RSS-enabled audio feeds via Apple's software (see Figure 1). As one tech journalist recalled, "prior to the iTunes 4.9 update on June 28, 2005, podcasts were so clumsily arranged around the internet and so technologically challenging to use on any device other than a desktop or laptop computer that only the most tech-savvy even knew they existed" (Friess, 2015). Apple's dominant market share in digital music sales (which reached $69 \%$ of the market by 2009) had the effect of instantly introducing podcasts to millions of potential listeners (Frommer, 2009). In essence, "iTunes 4.9 effectively brought podcasting

into

the cultural mainstream" (Bottomley, 2015, p. 164).

The structure of Apple's initial podcatcher interface and governance structure shaped the development of podcasting distribution in several important ways. On the surface, the iTunes version 4.9 podcatcher software interface was virtually identical to that of iTunes' music store, with a search bar interface to facilitate keyword searches, a list of "Top Podcasts" indicating popular or most downloaded shows, and thematic categories of podcast content. To harmonize the podcasts section of iTunes with its music store counterpart (which featured music album covers), Apple introduced cover art for their podcasts. This encouraged producers to create visually stimulating identifiers for podcasts (all others were given a generic RSS icon), which, in turn, shaped consumers' expectations for podcasts. In his keynote address announcing iTunes Version 4.9, Steve Jobs' enthusiasm for podcasting was specifically linked to larger companies' content (including major radio

Figure 1. Apple’s initial podcatcher software in June, 2005 (iTunes, version 4.9)

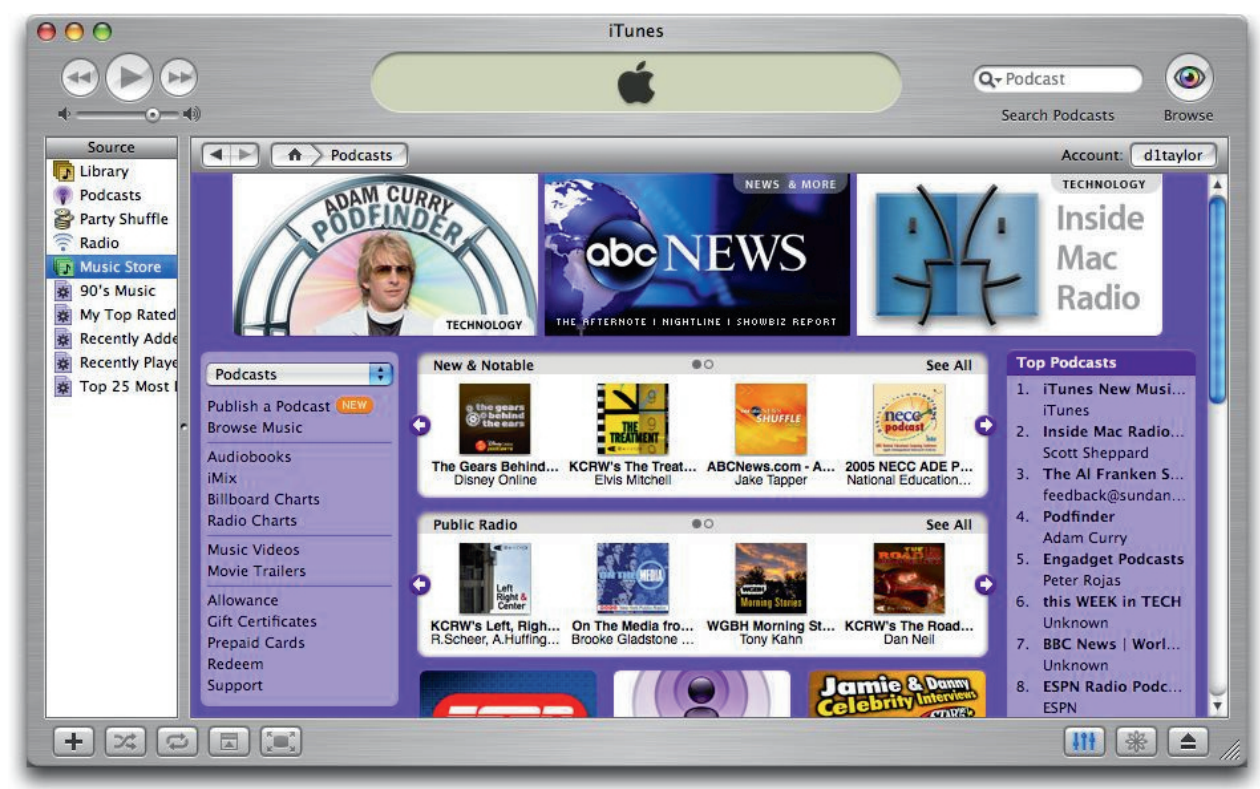

Image used with permission of AskDaveTaylor.com. 
broadcasters, network broadcasters, magazines, newspapers, and companies like Disney, Proctor \& Gamble, Ford, and General Motors) rather than amateurs. Content from these institutional content providers was given prominent space on iTunes which allowed consumers to discover it more readily. This linkage created by Steve Jobs and Apple between podcasting and professionalized, corporate media - bears more than a passing resemblance to David Sarnoff's RCA in the early days of radio. In the late 1920 s, RCA effectively defined the practice of radio broadcasting as a corporate one-to-many experience, rather than an audio exchange among everyday citizens or creative amateurs (see Sterne, et. al., 2008). Lastly, unlike its music store counterpart, Apple's pass-through of RSS feeds meant that all podcasts were distributed for free on iTunes. Since Apple did not choose to host the audio data files for download (thereby essentially adopting the decentralization model of RSS), it also thereby rendered paywalls, pay-perdownload, or other monetary exchanges for podcasting impossible via iTunes. This was one of the factors that pushed early podcasters to pursue advertiser-supported revenue models. Apple's iTunes was the first service to offer what scholars note as a "platform" service for podcasting, in that it provided a centralized repository of podcasts for ease of discovery, while also enabling podcasters to easily reach audiences by bundling their respective RSS feeds into a seamless digital interface.

\section{Podcasting's Post-2005 Platform Proliferation}

The first element of Nieborg and Poell's (2018) model concerns the impacts of platform infrastructure on market structures. In the case of podcasting, the decentralized infrastructure of RSS is largely responsible for its fragmented structure today. Since 2005, there has been a proliferation of podcast platforms. While this level of fragmentation would seem to defy the centripetal pull of platformization, recent moves toward greater centralization are slowly altering the status quo. There are three primary functions of media-related platform services: storage, discovery, and consumption. Typical Web 2.0 platforms such as YouTube, Flickr, Netflix, or Amazon Prime encapsulate all three of these functions: they present content for users to discover or search through their interface; they serve as a data repository for the files to be delivered to the user (whether via download or streaming); and they offer embedded playing software to allow users to consume media. Other types of interactions are made possible by platforms, of course, such as sociality, branding, and advertising, but I argue that storage, discovery, and consumption are the core functions without which content platforms services are incomplete. In the case of podcasting, these three functions have been often (though not always) separated into different services. As the primary distribution mechanism, RSS is simply a text file that points to content housed elsewhere on the web. Consequently, directory services like Apple Podcasts (Apple's new name for its iTunes podcatcher) point to the content rather than storing the audio or video files. Podcast data is typically stored on a traditional web host or on a dedicated podcast host site. Second, the content discovery function in podcasting is typically served by podcast directories that organize podcasts into categories along with keyword search capability. Third, listeners must 
rely upon software to consume digital audio content. On the one hand, this separation of functions into separate services has lowered barriers for market entrants, creating a rush of new competitive services specializing in one or more of these functions. On the other hand, the rush of companies and entrepreneurs to enter the podcasting market has created a somewhat confusing and chaotic landscape for both consumers and podcasters. The drive to streamline the process of podcast discovery and consumption in order to maximize audience size (in order to capture advertising revenue) is one of the major driving forces behind the increasing platformization of the medium today. While these core functions are outlined separately below for the purposes of analysis, it's important to note that they are often less clearly demarcated in practice, with some podcast services encompassing more than one function. These three functions and the current podcast platformization trends found in each are briefly considered below.

\section{Storage Platforms and Consumption Metrics}

Podcasting requires that audio data files are stored on servers and hosting services. There are a plethora companies hosting data files, many of which cater to the specific needs of podcasters. Libsyn (short for "Liberated Syndication"), launched in 2004, was the first dedicated podcast web hosting company, followed in 2005 by Blubrry. These services typically offer podcasters an array of features in addition to the storage of audio files and the management of the podcast RSS feed. Blubrry, for example, developed a plugin entitled PowerPress in 2008 to allow its customers to more easily create a maintain an RSS feed from their WordPress webpages and blogs. Other podcast hosting services have developed similar WordPress plugins. As interest in podcasting has risen dramatically, there has been a rapid expansion in podcast hosting companies, all of them promising to streamline the process of storing podcast audio files, maintaining and validating podcast RSS feeds, and registering podcasts on the major directories (discussed below). These hosting companies include Podbean (launched 2006), Podomatic (launched 2008), BlogTalkRadio (launched 2008), Audioboom (launched 2009), Buzzsprout (launched 2009), Spreaker (launched 2009), Simplecast (launched 2013), Fireside.fm (launched 2015), and Castos (launched 2017), among many others. Like all forms of web hosting, there is a monthly subscription cost associated with podcast hosting (often graduated according to the number of downloads), so the distributed infrastructure of RSS ironically may create an initial financial barrier to new podcasters. ${ }^{3}$

Podcast hosting companies are typically the first point of contact with independent podcasters, and they are the strongest podcast evangelists, since their business depends upon increasing the number of new content producers. With the rising commercial potential of the medium, some consolidation has begun to among podcast hosts. In 2017, for instance, BlogTalkRadio merged with Spreaker, and recently both companies were bought out by Voxnest.

A recent entrant to podcasting, Anchor.fm, launched to fanfare at South by Southwest festival in 2016, has staked its business model on providing its users a free, "one stop" solution to podcasting (Shontell, 
2016). Anchor.fm enables users to record audio directly via a smartphone app, edit the file and add music via app, upload it to Anchor's servers, and distribute it directly to major directories like Apple Podcasts, Google Play Music, and Spotify, all for free. Anchor.fm generates income by embedding advertising across all of its userproduced podcast content. There has been exponential growth in the introduction of new podcasts in the last year, and much of that can be attributed to the success of Anchor in lowering the entry barrier to podcast production. As Figure 2 illustrates, despite its recent market entry, Anchor.fm hosts $9.4 \%$ of all podcast episodes listed on the Apple Podcasts directory, second only to Soundcloud, which also offers free file hosting and audio streaming.

Figure 2. Podcast media hosting services (all time)

\begin{tabular}{|l|c|}
\hline \multicolumn{1}{|c|}{ Hosting Service } & $\begin{array}{c}\text { I\% of all podcasts in } \\
\text { Apple Podcasts }\end{array}$ \\
\hline 1. soundcloud.com & $14.8 \%$ \\
\hline 2. anchor.fm & $9.4 \%$ \\
\hline 3. libsyn.com & $8.6 \%$ \\
\hline 4. podomatic.com & $6.9 \%$ \\
\hline 5. podbean.com & $4.7 \%$ \\
\hline 6. archive.org & $2.8 \%$ \\
\hline 7. spreaker.com & $2.3 \%$ \\
\hline 8. squarespace.com & $1.9 \%$ \\
\hline 9. radio.com & $1.9 \%$ \\
\hline 10. ivoox.com & $1.4 \%$ \\
\hline 11. blubrry.com & $1.4 \%$ \\
\hline 12. buzzsprout.com & $1.3 \%$ \\
\hline 13. Other & $21.0 \%$ \\
\hline
\end{tabular}

Source: Chartable.com. (2018, August 29). What's really the top podcast hosting service? Retrieved September 2, 2018, from https:// chartable.com/blog/whats-really-the-toppodcast-hosting-service
For context, it's important to note that Anchor.fm's share of podcasts eclipses Libsyn, which has a long history as one of the first companies to host podcast RSS feeds.

Hosting companies are key players in the podcast market because their platforms allow them to gather valuable data on content consumption. Specifically, when content directories (like Apple Podcasts) reference the RSS feed to signal the transfer of an audio file, the podcast host registers the download from its server and notes the distribution source for the download as well as the operating system (desktop, iOS or Android). Although podcast metrics are still in their infancy and there has been much debate among industry players about the appropriate consumption data, one key measure has been "downloads per episode" (DPE) as reported by the podcast host. In 2017, hosting companies, along with advertisers, ad agencies, podcast networks, and representatives from public radio, collaborated under the auspices of the Interactive Advertising Bureau (IAB) to create a set of podcast measurement standards (Interactive Advertising Bureau, 2017) that were based largely on the DPE standard. This move placed podcast hosts as the central platforms in the generation of an industry-wide standard for podcast consumption (Fleck, 2018). The file storage platforms, then, act as a service provider to both podcasters and advertisers, though in distinct ways.

\section{Discovery Platforms and the Threat of Podcast Enclosure}

Since audio files are scattered around the internet and locatable via their RSS feeds, the second major function of podcast platforms is to 
facilitate the discovery of those podcasts and to allow listeners to "subscribe", which then provides them updates when new content is added for a particular show. Network externalities play an outsized role in podcast discovery, since listeners gravitate to directories that allow for greatest ease of discovery and that have the most comprehensive directories (network externalities are also key for search engines, and account for the huge market advantage of Google, for example). Discovery platforms typically do not store the audio files on their servers, and instead pass through the MP3 file that is accessed from the podcast host server. Like the MP3 file itself, the RSS feed acts as a "container technology" (Sterne, 2006). The most dominant directory is Apple Podcasts (formerly iTunes), which has enjoyed a significant "first to market" advantage such that most podcast listeners in the United States today are accessing those shows via Apple's directory (Interactive Advertising Bureau, 2017). While there are over a hundred podcast directories online, there are only two other significant discovery platforms: Google Play Music and Spotify. Professional and indie podcasters are often bewildered at the panoply of distribution outlets. Most aim to include their shows in as many discovery platforms as possible to maximize audience traffic. This has fueled the growth in podcast hosting platforms that promise a "one stop" solution for distribution via the largest directories.

Nieborg and Poell's (2018) model draws attention to the re-alignment of technical infrastructures thanks to the expanded reach of platforms via algorithms, SDKs, and APIs. In the case of podcasting, Apple's dominance as a discovery platform is even greater thanks to its API, which extends its directory service beyond its own Apple Podcasts service. Popular mobile podcast consumption apps such as Overcast, Pocket Casts, Downcast, and Podcast Addict all utilize Apple's directory for listing podcasts by linking their apps to the Apple Podcasts API. Apple's terms of service also introduces a form of editorial control over the content and presentation of podcasts on its directory. Recently, podcast hosts have received complaints about their shows being "de-listed" from Apple Podcasts for violating its rules or terms of service. For example, Apple does not allow its name or the names of its products to be in any podcast title, it blocks long podcast titles, it prevents URL links from podcast show notes and even polices cover art images (Cochrane \& Greenlee, 2018). Apple Podcasts currently acts also as an archive for podcast shows, allowing listeners to discover shows that are no longer being actively produced (something that podcasters call "podfaded" shows). Some estimates are that perhaps only half of the more than 500 thousand podcasts listed on Apple Podcasts have posted new content in the past three months (Goldstein, 2018). If Apple were to automatically "de-list" podcasts that had not recently updated their content, for example, Apple Podcasts would look quite different and much content would become almost impossible for listeners to find. Podcasting's dependence on platform APIs like Apple's is similar to trends in other social media such as Facebook and Twitter, wherein upstream decisions by the platform about information distribution can have profound consequences for downstream services, apps (Kastrenakes, 2018), and even media scholars (Burgess \& Bruns, 2012).

Along with the power of discovery platforms to direct listeners' attention, they also have the potential to create artificial scarcities of content in order to maximize the potential for revenue. For 
example, the business press has been excitedly suggesting for some time that the "Netflix of podcasting" moment has arrived, whereby platforms serve up "premium" or "exclusive" audio content to listeners that requires a subscription fee (Nagy, 2015; Porch, 2018; Rowe, 2017), although some are more circumspect about this potential (Quah, 2018; Rosenblatt, 2018). Podcasters have largely approached the notion of "Netflix-ization" with scorn and derision (Fang, 2018). In their eyes, podcasting will never transform into a subscription-based service due to the open architecture of RSS: How can you take something that is freely distributed via RSS and essentially lock it up behind a paywall?

The reality is, however, that platform enclosure - the creation of "walled gardens" of content available only to monthly subscribers is slowly taking hold in podcasting. Ironically, indie podcasters themselves have paved the way for enclosure by adopting similar techniques for monetizing their own podcasts: that is, by offering "premium" content that is available only via a monthly subscription. Larger industry players are replicating these strategies in an attempt to either lure more customers to their platform, or to "lock in" existing customers by encouraging them to access podcast content via their existing service. How can platform enclosure take hold in a medium that is built upon the open standard of RSS? The answer is that major audio content platforms are slowly steering listeners away from RSS-delivered audio content and into premium content that is platform-exclusive. Existing podcast networks such as Howl Premium and giant Stitcher Premium, for example, offer listeners paywalled content that is otherwise not available to listeners on other discovery platforms. Luminary - a new venture-capital backed podcasting service that launched in early 2019 - offers only exclusive, paywalled podcast content by media celebrities such as Trevor Noah and NPR's Guy Raz and will not make the content available via RSS (Rottgers, 2019). Music streaming giant
Spotify has made perhaps the most aggressive moves in favor of platform enclosure. For example, it introduced three new podcasts in 2017, and these shows were only available on its streaming app, and not via RSS (Roettgers, 2017). Spotify went a step further in 2018 and secured a deal to exclusively distribute the Joe Budden Podcast, a popular hip-hop music podcast (Saponara, 2018). Spotify's deal with Joe Budden was noteworthy because it stipulated that new episodes of Budden's podcast would not be distributed via RSS, but would instead be found only on Spotify. Spotify's deal is the first that would essentially remove content that was previously distributed via RSS and lock its distribution into a proprietary platform. This move is not dissimilar to the recent strategic alliance between Facebook and the New York Times, where readers increasingly link to online news via the social media giant at the expense of the open framework of RSS (Plantin et al., 2018). Finally, in February 2019, Spotify stunned the podcasting industry by announcing its $\$ 337$ million acquisition of Gimlet Media - the podcast production company co-founded in 2014 by NPR veteran Alex Blumberg - and Anchor.fm, the fastest-growing podcasting platform (Spangler, 2019; Szalai, 2019). Spotify's aim is likely to capitalize on the high production values and audiences for Gimlet's podcasts by bringing its listeners to Spotify's platform and away from other discovery platforms like Apple Podcasts and Google Podcasts. As podcasting becomes less dependent on the open RSS standard for distribution (See Figure 3), podcast directories and streaming platforms are aiming to shift distribution away from open infrastructures and toward their own services to maximize the "winner take all" functions of platforms. In 


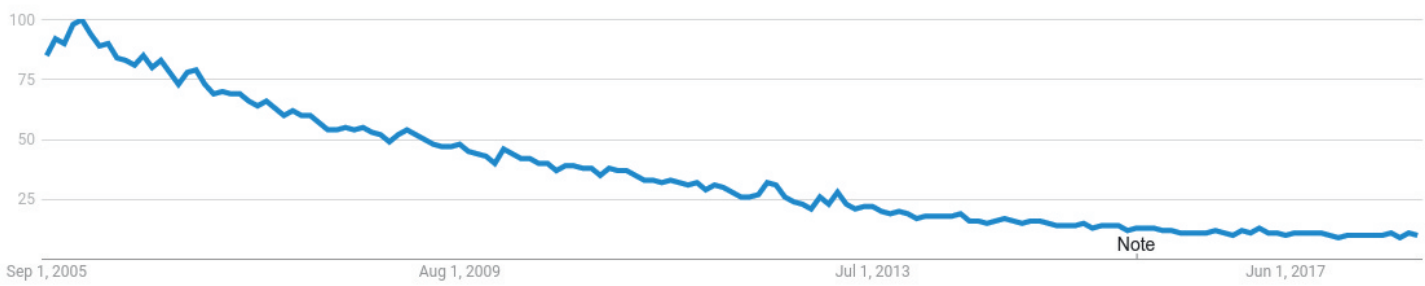

other words, once RSS is no longer required to distribute podcasts, the threat of platform enclosure will only increase.

\section{Consumption Apps and Platform Alliances}

The third important function of online media platforms is to allow users to consume the content. In the early years of podcasting prior to the introduction of the iPhone in 2007, most listeners consumed podcasts either on their computers or on dedicated digital audio devices such as iPods. In an increasingly mobile era, however, podcast consumption has shifted to mobile devices, mediated largely via the iOS and Android mobile operating systems. The Interactive Advertising Bureau (2017) found that roughly $45-52 \%$ of all podcast consumption in the United States was achieved via the Apple Podcast app on iOS. The inclusion of a default, pre-installed podcast app in Apple's iOS Version 8.0 update in 2014 was a significant factor in expanding the audience for podcasting, resulting in 13.7 billion episode downloads on that platform in 2017 alone (Locker, 2018). This dramatic increase in podcast listenership over such a short period of time demonstrates the power of apps - and the default software settings behind those apps - to drive discovery and consumption behaviors (for more on the power of software defaults, see Kitchin \& Dodge, 2011; Shah \& Kesan, 2008; Shah \& Sandvig, 2008). While Apple's iOS podcast app dominates, there are numerous competing apps that allow users to subscribe and consume podcasts such as Stitcher, Pocket Casts, Overcast, Podcast Addict, BeyondPod, DoggCatcher and Downcast, and Castbox, among many others. Adding to the fragmentation in consumption apps, some podcast hosting companies (like Podbean, for example) have launched a dedicated podcasting app. Lastly, the app-ification of podcasting is also well underway, with a number of popular podcasts such as This American Life and The Dave Ramsey Show launching their own dedicated apps within the iOS and Android app stores. Podcast hosting companies like Libsyn and Spreaker have fueled app-ification by offering to launch a dedicated app for their customers' podcasts as part of their subscription fee.

Mobile consumption apps are critical to the infrastructure of podcasting because they provide a dual functionality as both tools of content discovery and consumption. Many of these apps are aligning themselves strategically and financially with discovery platforms in order to monetize their users' data and to introduce artificial scarcities. A number of mobile apps have been increasingly become the target of acquisitions by large media companies wishing to gain a foothold in the podcasting market. For example, E.W. Scripps, a traditional media company that owns a diverse portfolio of legacy media such as newspapers, 
broadcast radio and television stations, purchased podcast advertising firm Midroll in 2015 for \$10 million and quickly followed with a $\$ 4.5$ million purchase of podcasting app Stitcher in 2016 (Perlberg, 2016). Sensing the importance of consumption apps, in May 2018 a consortium of public radio organizations including NPR, WNYC Studios, WBEZ Chicago, and This American Life, purchased Pocket Casts (Mullin, 2018).

Podcast mobile apps offer their own forms of interactivity, sociability, and content curation by highlighting within the app a unique constellation of content specifically designed for users (Morris \& Patterson, 2015). This form of specialized curation has been increasingly influenced by strategic alliances among consumption apps and large content providers. For instance, Castbox - a new podcast mobile app that has already raised over $\$ 13$ million in venture capital funding - has a section of its free app designated as "premium" content that is available via an in-app subscription (Sawers, 2018). Some of the content in this premium section is produced by Castbox itself, and the rest is produced by its content partner, Wondery (InsideRadio, 2018). Similarly, the iHeartRadio app (which has a very small curated list of podcasts in its own directory of 20,000), has leveraged its ties to the broadcast radio industry to make aggressive moves into podcasting. iHeartRadio purchased the HowStuffWorks podcast network for $\$ 55$ million on September 15,2018 , enabling it to exclusively feature the those podcasts and paving the way for more "premium" content for its mobile app (Jarvey, 2018). While Apple has yet to move its own podcasting directory in the direction of platform enclosure, it is aggressively pursuing this strategy in video, having invested US \$1 billion in acquiring and commissioning original content for a new streaming service that will compete directly with other video platform giants such as Netflix, Amazon Prime, and Hulu (D’Alessandro, 2018; Mickle, 2017). These platform alliances indicate that the fragmentation in podcasting is slowly giving way to platform consolidation in the service of monetization and audience maximization.

\section{Platform Governance in a Fragmented Ecosystem}

Nieborg and Poell's (2018) model also draws attention to the means by which platform governance shapes the means of cultural production. As noted above, podcasting has seen a proliferation of storage, discovery, and consumption platforms since 2005, making governance a patchwork affair. Each of the storage and discovery platforms come with their own terms of service (TOS) and editorial guidelines. As Gillespie (2018, p. 263) has noted, the rules established by platforms exist primarily to protect the platform's public brand and profitability, though these goals are often intertwined with a "deeply felt commitment of the platform operators for nurturing a healthy community." As the leading discovery platform in the U.S., Apple Podcasts' TOS outlines content parameters that are similar to those of other online platforms. For example, Apple's terms of service prohibits the following from podcasts listed on their iTunes services:

- Irrelevant content or spam.

- Explicit language without setting the $<$ explicit> tag.

- Content that could be construed as racist, misogynist, or homophobic.

- Explicit or self-censored explicit language in titles, subtitles, or descriptions.

- References to illegal drugs, profanity, or violence in the title, description, artwork, or episodes.

- Content depicting graphic sex, violence, gore, illegal drugs, or hate themes. 
- Third-party content or trademarks without legal authorization or usage rights.

Source: Apple Media Services Terms and Conditions (https://www.apple.com/legal/internet-services/ itunes/us/terms.html)

Apple's governance structure goes beyond the above editorial guidelines to include the protection of its brand identity. For instance, Apple prohibits the words Apple Music, iTunes Store, iTunes, Apple Podcasts, or Apple Inc. in podcast titles or descriptions, and prohibits pictures of its corporate logos, or of its technology (such as iPhone, iPad, or iPod) in any cover art image in its podcast directory. Podcast hosting companies have similar, but distinct, TOS agreements listed on their services, but given the increase in podcast launches post2014 and their relatively small staffs, these services do not closely monitor the content of the podcasts they host.

New podcast host entrant Anchor.fm has caused controversy within the podcasting community over its TOS. Flush with $\$ 10$ million in venture capital funds (Roof, 2017), Anchor has offered itself to would-be podcasters as a free service. Veteran podcasters and podcast hosting companies immediately began scrutinizing Anchor's TOS and found that the users of the site "grant [them] a worldwide, non-exclusive, royalty-free, sublicensable and transferable license to use, edit, modify, aggregate, reproduce, distribute, prepare derivative works of, display, and perform the User Content in connection with the operation of the Services." As well-known podcaster Dave Jackson (2018) has noted, Anchor's claim of ownership of user-generated content are much more restrictive than comparative social media platforms, including those of Twitter and Facebook. As the fastest growing site for podcast hosting, Anchor's move to centralize the control of the rights to podcast content points to the power of platforms to offer universal access as a "loss leader" in order to monetize user-generated content, in much the same way as Google leverages its content to sell advertising on YouTube.

The fragmented nature of podcast platforms also makes for a patchwork enforcement of editorial guidelines. In 2018, controversy surrounding the content of radio host and conspiracy theorist Alex Jones' Infowars program epitomized the challenges in policing content across podcasting's many platforms. On his Infowars radio program, which is also released as a podcast, along with several other Infowars-branded podcasts, right-wing host Jones was wellknown for trafficking in conspiracy theories, including one that the Sandy Hook shooting was staged by "crisis actors." On August 5, 2018, after complaints from Sandy Hook victims' families and listeners, Spotify removed several episodes of Jones' podcast for violating its "hate speech" policy, followed swiftly by Apple, which completely removed (de-listed) five of its six Inforwars-branded podcasts from its service (Vernon, 2018). Podcast host Spreaker, which stored the audio files for The Alex Jones Show podcast, found itself suddenly thrust into the midst of a public controversy. Citing its own terms of service which prohibited the publication of "any content that promotes, either directly or indirectly, hate, racism, discrimination, pornography, or violence," Spreaker removed Jones' content from its own servers following the moves by Spotify and Apple (Schneider, 2018). Many podcasters took note, however, that the main Infowars RSS feed was self-hosted on Jones' own website, and that listeners 
who wanted to access the content could simply subscribe manually to the feed (as they had done in the early days of podcasting) and continue to receive new episodes. Additionally, Apple faced mounting criticism for allowing the Infowars app (which contained much if not all of the same content) to remain in its App Store, arguing that it had not violated its App Store TOS, which did not feature the same language explicitly banning forms of hate speech. After a month of heavy criticism, on September 7, 2018 Apple relented and removed the Infowars app as well (Nicas, 2018). Apple's choice to delist Infowars also laid bare the symbiotic entanglements between their podcast directory and other apps, since all consumption apps that relied on Apple's directory API saw Alex Jones' content immediately removed from their directories as well, effectively ceding editorial control to Apple. The Infowars debacle served as an important inflection point in podcasting because it demonstrated the difficulties in managing a fractured governance structure from a multi-platform ecosystem.

\section{Conclusion}

Podcasting is in the midst of a transition. Online platforms have begun making major investments in the medium, hoping to attract listeners, advertisers, and new podcasters. Thanks to the decentralized architecture of RSS, the process of platformization outlined by Nieborg and Poell (2018) is progressing somewhat differently than for other large content-sharing services. Since the three functions of content platforms - storage, discovery, and consumption - are dispersed among podcast hosting companies, directories like Apple Podcasts, and mobile apps, respectively, it would appear that podcasting is less susceptible to the forces of platformization. However, as this discussion has outlined, the podcast market is undergoing rapid transformation, spurred largely by the interest of large tech giants like Apple, Spotify, and Google. Strategic alliances among discovery and consumption platforms, in particular, are moving podcasting in the direction of fully integrated content platforms like YouTube, Facebook, and Twitter. As Srnicek (2016, p. 98) has noted, the "tendencies" that emerge from the competitive dynamics of large platforms include the "expansion of extraction, positioning as a gatekeeper, and enclosure of ecosystems." Each of these tendencies can be observed in podcasting today, at least in the United States. While major U.S. internet companies Apple and Google have already begun pushing podcasting in the direction of platformization, recent strategic acquisitions by Swedish audio giant Spotify point to a more platform-centric future worldwide thanks to Spotify's global reach.

The network effects associated with platformization have differential benefits for listeners, content producers, podcast platforms, and advertisers. As podcasting becomes more popular, the number of podcasts in production has vastly expanded, creating an increasingly crowded content landscape. This presents a challenge for listeners hoping to discover new shows that may appeal to their particular interests. For listeners, then, centralized repositories like Apple Podcasts, Spotify, or Google Play carry positive network effects thanks to the ease of discovery and the crowdsourced curation of podcasts (much like the Rotten Tomatoes ratings for films or Amazon's user-generated product reviews). Podcast networks, distributors and advertisers, on 
the other hand, derive much different benefits from the network effects of platform centralization. For these players, the major value of platformization comes from their ability to glean more accurate consumption data from a large audience for the purposes of monetization. Current audience consumption data are based chiefly on server-side measures (downloads per episode) and platform-side measures such as subscriptions via a podcatchers like Apple Podcasts, Spotify, Castbox, and Overcast, among others. What centralized platforms like Apple Podcasts and Spotify offer to distributors and advertisers is a glimpse into actual audience listening consumption. How much of that podcast episode, for example, was actually heard by audiences, and at what point did large numbers of listeners abandon the episode? Moreover, who is actually listening to what podcast?

Thanks to the dominance of Apple's mobile iOS platform, at least in the United States (Perez, 2018), Apple has access to a vast trove of personal data, including valuable information such as names, addresses, age, race, gender, credit card numbers, and more. While Apple agreed to release more specific consumption data to podcast producers and publishers in late 2017 (Webster, 2017), it chose to anonymize the audience data, thus preventing would-be podcast advertisers from employing target marketing campaigns with the kind of precision that they routinely employ on social media platforms (Willens, 2017). Choosing to protect user-level audience data and essentially give away anonymized podcast consumption statistics may serve to further cement Apple's dominance in the podcast ecosystem. Since the value of these data will only increase as more and more audiences access their podcasts via Apple's iOS platform (or via the Apple Podcasts' API), Apple is likely seeking to retain its long-held position as the industry leader. Spotify's recent acquisitions of Anchor and Gimlet Media present a serious challenge to Apple's long-held platform dominance in podcasting, however. If Spotify eschews anonymization in its metrics and links its podcast audience consumption data to specific subscriber accounts - including credit card data and demographics, for example - it could offer a stronger value proposition for podcast networks and advertisers keen on monetizing via target marketing.

The implications of platform consolidation for independent podcasters are potentially more negative. On the one hand, the proliferation of storage and discovery platforms today works to the benefit of independent podcasters and amateurs because the ecosystem is still in considerable flux. Competing services and new technologies are launching almost daily, which lowers barriers to entry for new producers, particularly in the case of free services like Anchor.fm and Soundcloud. Once this industry churn has settled and the pace of consolidation quickens, distribution options for podcasters may begin to dwindle, leaving them with less autonomy to transform their hobby into full-time work on their own terms. Once large market players like Apple, Google, Amazon, and Spotify begin leveraging the power of network externalities to expand the podcast audience by offering paid subscriptionbased podcast content to their large existing customer bases, professionalquality podcasting (sometimes called "procasting") will become inextricably linked to platform services. As Nieborg and Poell (2018, p. 3) note, "as cultural production is becoming increasingly 
platform dependent, the autonomy and economic sustainability of particular forms of cultural production is increasingly compromised." Content producers wishing to reach audiences will naturally gravitate to such services because of their large listener base and the promotional advantages they provide. Independent podcasters have publicly scoffed at the idea that these platforms will ever dominate the medium because of its reliance on an open web standard, but content exclusivity deals like the one Spotify has struck with the Joe Budden Podcast, its acquisitions of Gimlet Media and Anchor, and the increasing consolidation among discovery and consumption platforms may point to a platformcentric future, leaving RSS as a "second-class" distribution mechanism reserved for amateurs. In this sense, the oligopolization of podcast discovery stands to reshape the medium in its second decade.

\section{References}

Berry, R. (2006). Will the iPod Kill the Radio Star? Profiling Podcasting as Radio. Convergence: The International Journal of Research into New Media Technologies, 12(2), 143-162.

Blubrry. (2017, June 14). Podcast Statistics 2017: Will Android Continue To Gain Market Share While Apple Slips? Retrieved May 8, 2018, from https:// powerpresspodcast.com/2017/06/14/ podcast-statistics-ns-2017/

Bonini, T. (2015). The "Second Age" of podcasting: Reframing podcasting as a new digital mass medium. Quaderns Del CAC, 41(18), 21-30.

Bottomley, A. J. (2015). Podcasting: A Decade in the Life of a "New" Audio Medium: Introduction. Journal of Radio \& Audio Media, 22(2), 164-169. https://doi.org/ 10.1080/19376529.2015.1082880
Bottomley, A. J. (2016). Internet radio: a history of a medium in transition (Dissertation). University of Wisconsin-Madison, Madison, Wis.

Burgess, J., \& Bruns, A. (2012). Twitter Archives and the Challenges of "Big Social Data" for Media and Communication Research. M/C Journal, 15(5). Retrieved from http:// journal.media-culture.org.au/ index.php/mcjournal/article/view/ 561

Cochrane, T. (2005a). History of podcasting. Retrieved July 30, 2018, from https://create.blubrry.com/ manual/about-podcasting/history-ofpodcasting-new/

Cochrane, T. (2005b). Podcasting: Do-ItYourself Guide. Indianapolis, IN: Wiley.

Cochrane, T., \& Greenlee, R. (2018, September 9). Apple Editorial Control \#218. New Media Show. Retrieved from https://newmediashow.com

D’Alessandro, A. (2018, September 9). Apple Acquires Global Rights To Documentary 'The Elephant Queen.' Deadline. Retrieved from https:// deadline.com/2018/09/appleacquires-global-rights-todocumentary-the-elephant-queentoronto-international-film-festival1202460419/

Edison Research. (2018). The Infinite Dial 2018. Retrieved from http:// www.edisonresearch.com/infinitedial-2018/

Fang, W. (2018, June 4). The Freedom of Podcasting and Netflix for podcasts won't work. Retrieved September 10, 2018, from https:// broadcast.listennotes.com/thefreedom-of-podcasting-and-netflixfor-podcasts-wont-work- 
50fde042c915

Fleck, A. (2018, June 21). Podcast Metrics Are

Still the Wild West: But Networks Are

Moving to Change That. Adweek. Retrieved

from https://www.adweek.com/digital/

podcast-metrics-are-still-the-wild-west-

but-networks-are-moving-to-change-that/

Friess, S. (2015, July 1). The media milestone the media (fittingly?) forgot. Retrieved July 19, 2018, from https://www.cjr.org/ analysis/podcast_milestone.php

Frommer, D. (2009, August 18). iTunes Now 25\% Of All Music Sold In U.S. Business Insider. Retrieved from https:// www. businessinsider.com/itunes-now-25of-all-music-sold-2009-8

Gillespie, T. (2018). Governance of and by platforms. In J. Burgess, A. E. Marwick, \& T. Poell (Eds.), The SAGE Handbook of Social Media (pp. 254-278). Thousand Oaks, CA: SAGE Publications.

Goldstein, S. (2018, August 22). How Many of the 540,000 Podcasts Have "Podfaded?" Retrieved September 1, 2018, from https:// www.amplifimedia.com/blogstein/ 2018/8/22/how-many-of-the-550000podcasts-are-actually-active

Hammersley, B. (2003). Content Syndication with RSS (1st edition). Beijing: Farnham: O’Reilly Media.

Hammersley, B. (2004, February 11). Audible revolution. The Guardian. Retrieved from https://www.theguardian.com/media/2004/ feb/12/broadcasting.digitalmedia

InsideRadio. (2018, June 29). Castbox Launches Subscription Model For Podcast Publishers. Retrieved September 10, 2018, from http://www.insideradio.com/free/ castbox-launches-subscription-model-forpodcast-publishers/article_65c561c07b61-11e8-8967-23ebebdc9704.html Interactive Advertising Bureau. (2017). IAB Podcast Measurement Technical
Guidelines, Version 2.0. IAB Tech

Lab. Retrieved from https:// www.iab.com/wp-content/uploads/ 2017/12/

Podcast_Measurement_v2-FinalDec2017.pdf

Jackson, D. (2018, August 12). Anchor.fm Terms of Service Compared to Other Media Hosts. Retrieved September 16, 2018, from http:// davidjackson.org/anchor-fm-termsof-service-compared-to-othermedia-hosts/

Jarvey, N. (2018, September 13). iHeartMedia to Acquire Podcasting Company Stuff Media. The Hollywood Reporter. Retrieved from https:// www.hollywoodreporter.com/news/ iheartmedia-acquire-podcastingcompany-stuff-media-1143014 Kafka, P. (2017, December 14). Apple has finally turned on its podcast analytics feature. Retrieved May 12, 2018, from https://www.recode.net/ 2017/12/14/16778268/applepodcast-find-listens-downloadsanalytics-available

Kastrenakes, J. (2018, May 16). Twitter is going to make third-party apps worse starting in August. Retrieved September 16, 2018, from https:// www.theverge.com/ 2018/5/16/17362138/twitter-apithird-party-apps-changes-explained

Kitchin, R., \& Dodge, M. (2011). Code/ space software and everyday life. Cambridge, Mass.: MIT Press.

Locker, M. (2018, April 25). Apple's podcasts just topped 50 billion alltime downloads and streams. Fast Company. Retrieved from https:// www.fastcompany.com/40563318/ apples-podcasts-just-topped-50- 
billion-all-time-downloads-and-streams

Mallenbaum, C. (2015, April 13). The "Serial

effect” hasn't worn off. USA Today.

Retrieved from https://www.usatoday.com/ story/life/2015/04/13/serial-podcastundisclosed/25501075/

Markman, K. M., \& Sawyer, C. E. (2014). Why Pod? Further Explorations of the Motivations for Independent Podcasting. Journal of Radio \& Audio Media, 21(1), $20-$ 35. https://doi.org/ 10.1080/19376529.2014.891211

Mickle, T. (2017, August 16). Apple Readies \$1 Billion War Chest for Hollywood Programming. Wall Street Journal. Retrieved from https://www.wsj.com/ articles/apple-readies-1-billion-war-chestfor-hollywood-programming-1502874004

Morris, J. W., \& Patterson, E. (2015). Podcasting and its Apps: Software, Sound, and the Interfaces of Digital Audio. Journal of Radio \& Audio Media, 22(2), 220-230. https://doi.org/ 10.1080/19376529.2015.1083374

Mullin, B. (2018, May 3). Public Radio Organizations Buy Pocket Casts. Wall Street Journal. Retrieved from https:// www.wsj.com/articles/public-radioorganizations-buy-pocket-casts1525366680

Nagy, E. (2015, August 17). Is Howl The "Netflix Of Podcasts" We've Been Waiting For? Retrieved September 1, 2018, from https:// www.fastcompany.com/3049750/is-howlthe-netflix-of-podcasts-weve-beenwaiting-for

Nicas, J. (2018, September 9). Alex Jones's Infowars Is Removed From Apple's App Store. The New York Times. Retrieved from https://www.nytimes.com/2018/09/07/ business/infowars-app-alex-jones-appleban.html

Nieborg, D. B., \& Poell, T. (2018). The platformization of cultural production: Theorizing the contingent cultural commodity. New Media \& Society, 1-18. https://doi.org/

10.1177/1461444818769694

Perez, S. (2018, August 8). Facebook is now a major mobile browser in U.S., with $10 \%+$ market share in many states. Retrieved August 6, 2018, from http://social.techcrunch.com/ 2018/08/06/facebook-is-now-amajor-mobile-browser-in-u-s-with10-market-share-in-many-states/

Perlberg, S. (2016, June 6). E.W. Scripps Buys Podcast Company Stitcher. Wall Street Journal. Retrieved from http:// www.wsj.com/articles/e-w-scrippsbuys-podcast-company-stitcher1465239600

Plantin, J.-C., Lagoze, C., Edwards, P. N., \& Sandvig, C. (2018). Infrastructure studies meet platform studies in the age of Google and Facebook. New Media \& Society, 20(1), 293-310. https://doi.org/ $10.1177 / 1461444816661553$

Porch, S. (2018, June 27). Is the "Netflix of podcasts" moment finally here? Retrieved September 2, 2018, from https://www.fastcompany.com/ 40589660/these-companies-aretrying-to-build-the-netflix-ofpodcasts

Quah, N. (2018, July 3). Enough with the "Netflix for audio." Podcast companies should take a cue from meditation apps instead. Retrieved September 10, 2018, from http:// www.niemanlab.org/2018/07/ enough-with-the-netflix-for-audiopodcast-companies-should-take-acue-from-meditation-apps-instead/

Roettgers, J. (2017, February 23). Spotify Launches Three New Original Podcasts. Retrieved September 2, 2018, from https://variety.com/2017/ digital/news/spotify-originalpodcasts-1201994875/

Roof, K. (2017, September 28). Anchor 
raises $\$ 10$ million for podcast platform.

Retrieved September 16, 2018, from http://

social.techcrunch.com/2017/09/28/

anchor-raises-10-million-for-podcast-

platform/

Rosenblatt, B. (2018, March 23). "Netflix For

Podcasts" Looks Doubtful Despite Steadily

Growing Listenership. Retrieved

September 1, 2018, from https://

www.forbes.com/sites/billrosenblatt/

2018/03/23/podcast-listenership-

continues-to-grow-but-revenue-growth-

may-be-challenging/

Rottgers, J. (2019, March 4). Luminary Podcast

Subscription Service to Launch With

Exclusive Shows From Lena Dunham,

Trevor Noah. Variety. Retrieved from https://

variety.com/2019/digital/news/luminary-

podcast-subscription-service-

1203154250/

Rowe, A. (2017, May 5). Why the "Netflix of

Podcasts" Is the Biggest Media

Battleground of 2017. Retrieved

September 1, 2018, from https://tech.co/

netflix-podcasts-biggest-media-

battleground-2017-2017-05

Saponara, M. (2018, August 22). Joe Budden's

“The Joe Budden Podcast" Lands

Partnership With Spotify. Billboard.

Retrieved from https://www.billboard.com/

articles/columns/hip-hop/8471655/joe-

budden-joe-budden-podcast-spotify-deal

Sawers, P. (2018, April 25). Castbox raises

$\$ 13.5$ million as podcast investment boom continues. VentureBeat. Retrieved from https://venturebeat.com/2018/04/25/ castbox-raises-13-5-million-as-podcastinvestment-boom-continues/

Schneider, M. (2018, August 6). Apple and Spotify Remove Alex Jones' Podcast; Other InfoWars Shows Remain. Billboard.

Retrieved from https://www.billboard.com/ articles/business/8468722/apple-spotifyremove-alex-jones-podcast-infowarsshows

Shah, R. C., \& Kesan, J. P. (2008). Setting Online
Policy with Software Defaults.

Information, Communication \&

Society, 11(7), 989-1007. https://

doi.org/

10.1080/13691180802109097

Shah, R. C., \& Sandvig, C. (2008). Software

Defaults as de facto Regulation: The

Case of the Wireless Internet.

Information, Communication \&

Society, 11(1), 25-46. https://doi.org/

10.1080/13691180701858836

Shontell, A. (2016, March 16). Investors

can't stop talking about a one-

month-old app called Anchor.

Retrieved September 2, 2018, from

https://www.businessinsider.com/

what-is-anchor-fm-and-how-to-use-

the-app-2016-3

Spangler, T. (2019, February 15). Spotify

Paid Nearly $\$ 340$ Million to Buy

Podcast Startups Gimlet and Anchor.

Variety. Retrieved from https://

www.iheart.com/playlist/variety-

power-of-women-2019-

312064750-

EvupBB7c7woZYHEkWHpV79/

Srnicek, N. (2016). Platform Capitalism. Cambridge, UK: Polity.

Sterne, J. (2006). The mp3 as cultural artifact. New Media \& Society, 8(5), 825-842.

Sterne, J., Morris, J., Baker, M. B., \& Freire, A. M. (2008). The Politics of

Podcasting. The Fibreculture Journal, (13). Retrieved from http:// thirteen.fibreculturejournal.org/fcj087-the-politics-of-podcasting/

Szalai, G. (2019, February 6). Spotify to Acquire Podcast Firms Gimlet Media, Anchor. Billboard. Retrieved from https://www.billboard.com/articles/ business/radio/8496801/spotifyacquire-podcast-firms-gimletmedia-anchor

Vernon, P. (2018, August 7). Alex Jones

forces tech giants to act like media 
companies. Columbia Journalism Review.

Retrieved from https://www.cjr.org/

the_media_today/infowars-facebook-

youtube.php

Webster, T. (2017, June 18). The Partially-Filled

Glass of Apple's New Podcast Statistics.

Retrieved May 9, 2018, from https://

medium.com/@webby2001/the-partially-

filled-glass-of-apples-new-podcast-

statistics-7bc8e273bf9b

Wikipedia. (2017). History of podcasting. In

Wikipedia. Retrieved from https://

en.wikipedia.org/w/index.php?

title=History_of_podcasting\&oldid=76435

3357

Willens, M. (2017, June 12). What you need to know about Apple's new podcast analytics. Retrieved August 6, 2018, from https:// digiday.com/media/need-know-applesnew-podcast-analytics/ 